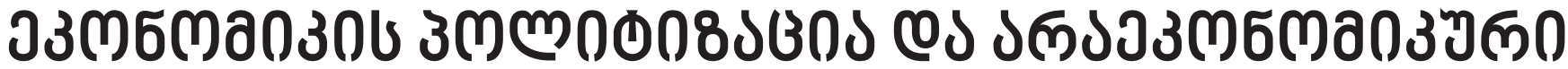

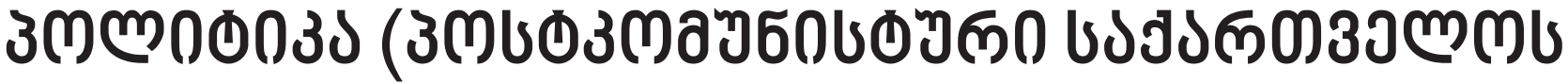

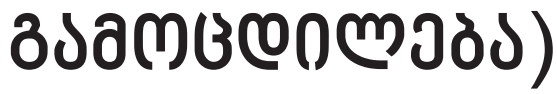

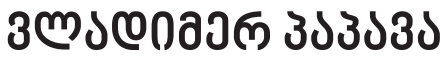

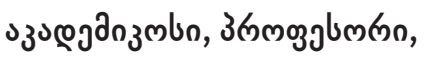

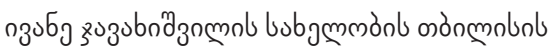

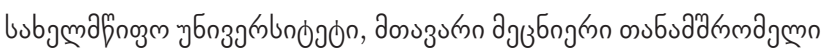

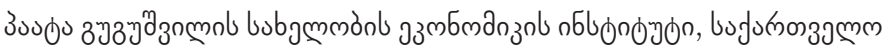

vladimer.papava@tsu.ge

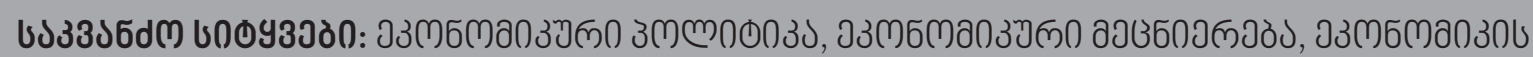

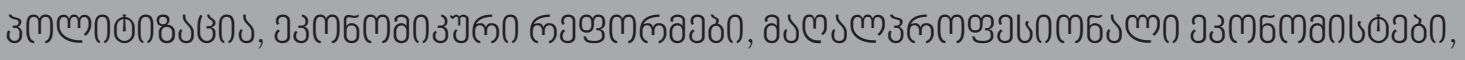

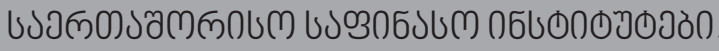

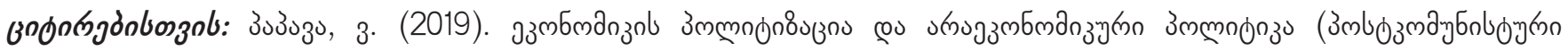

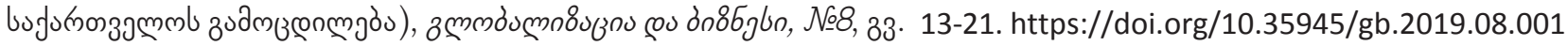

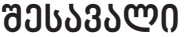

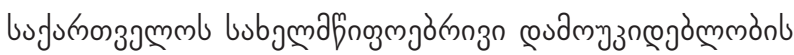

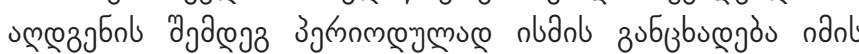

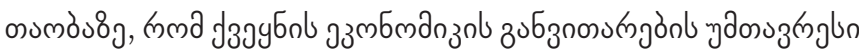

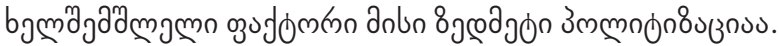

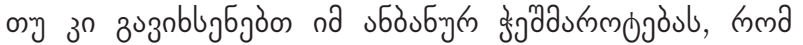

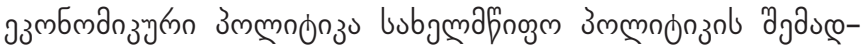

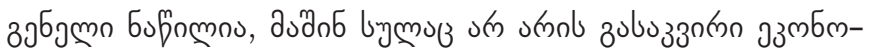

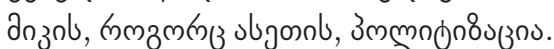

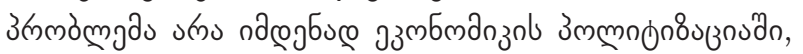

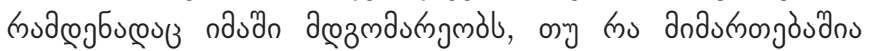

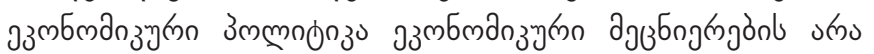

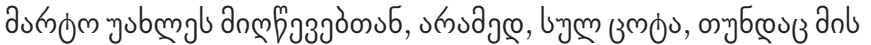

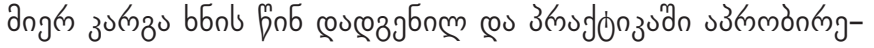

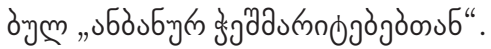

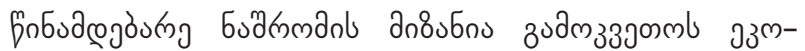

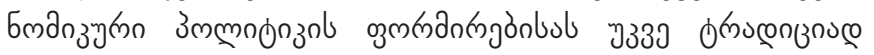

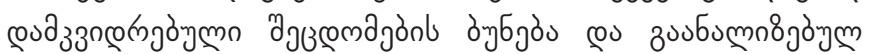

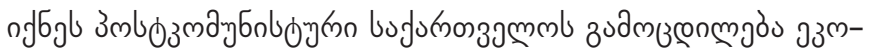

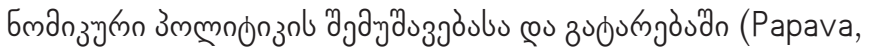

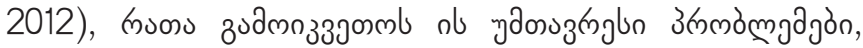

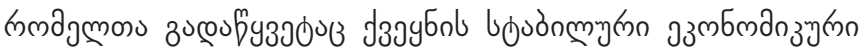

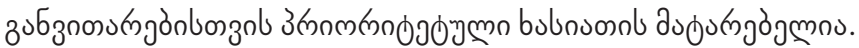

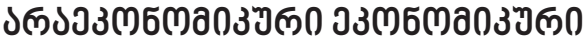

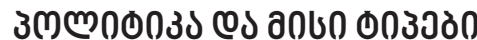

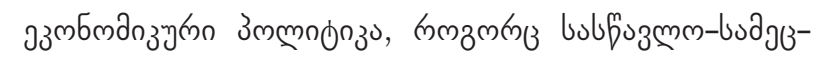

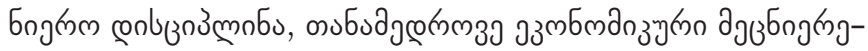

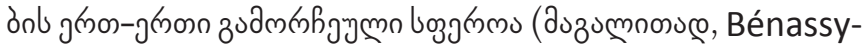
Quéré, Coeuré, and etc., 2016).

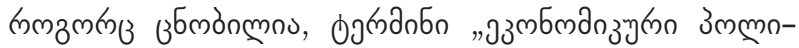

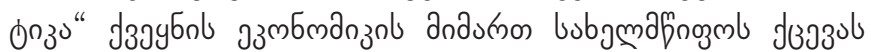

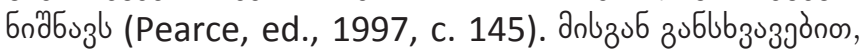

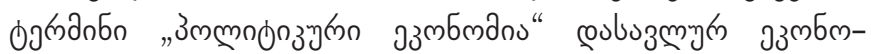

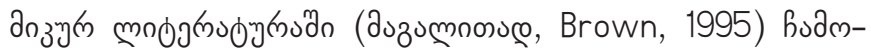

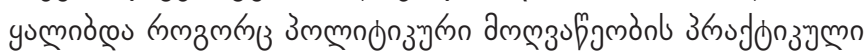

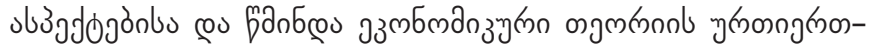

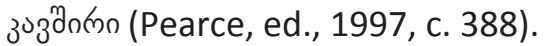

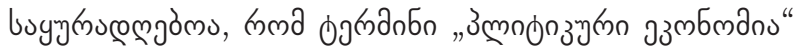

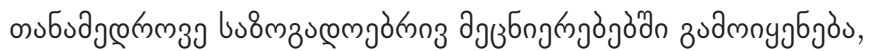

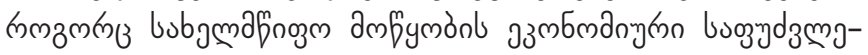

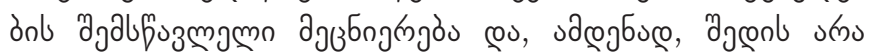

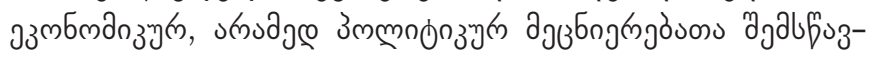

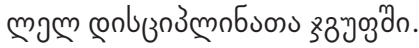

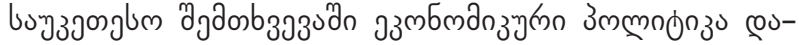
оुचdб

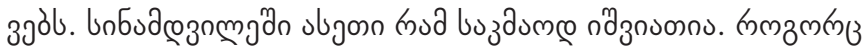




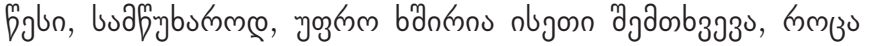

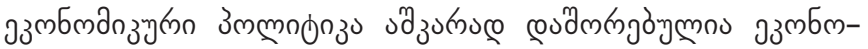

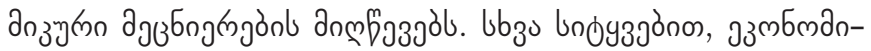

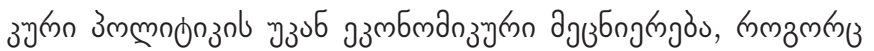

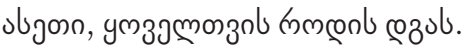

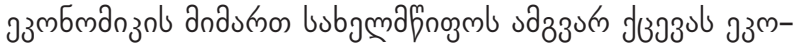

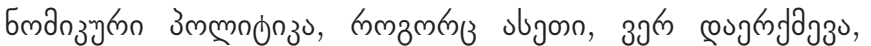

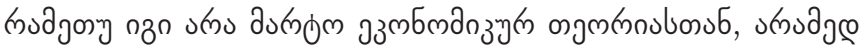

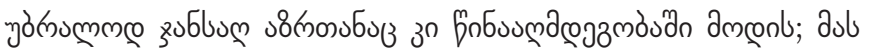

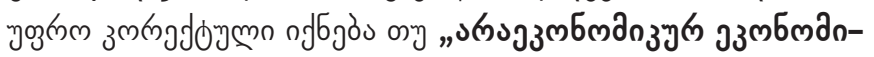

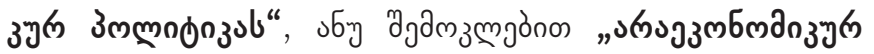

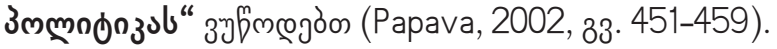

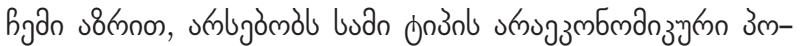

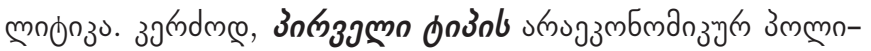
фnzul buдn зuбalus

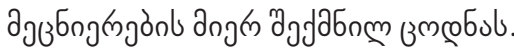

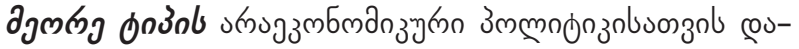

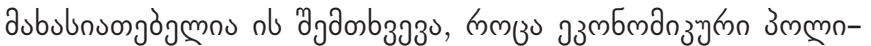

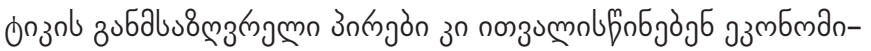

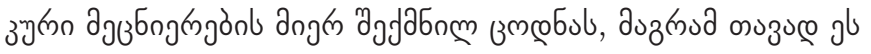

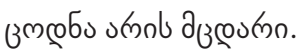

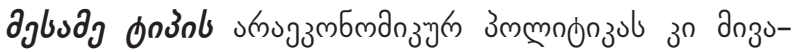

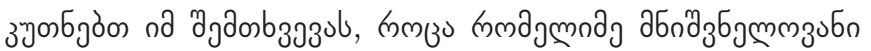

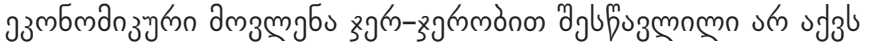

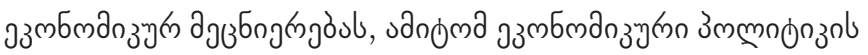

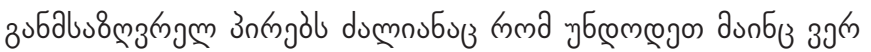

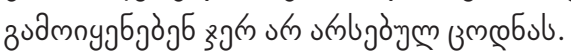

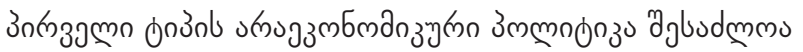

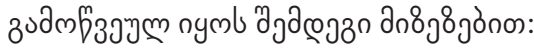

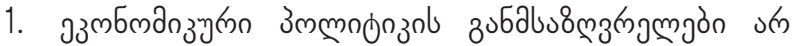

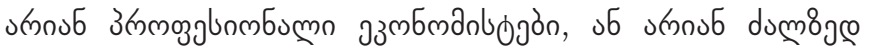

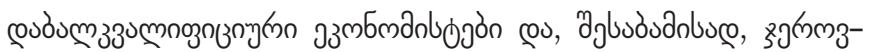

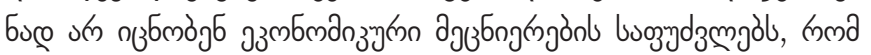

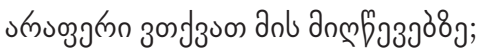

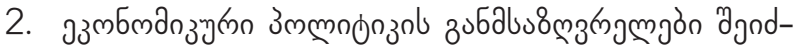

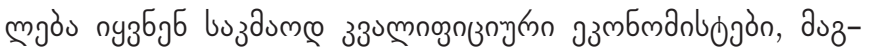

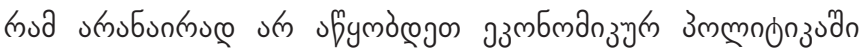

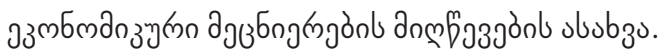

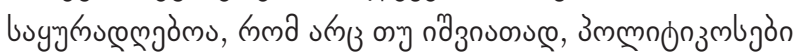

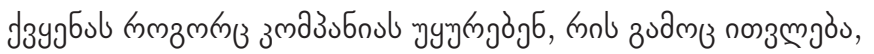

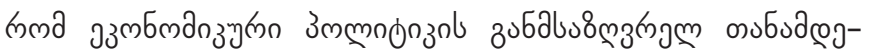

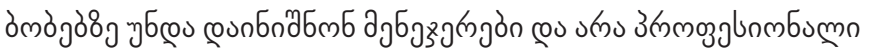

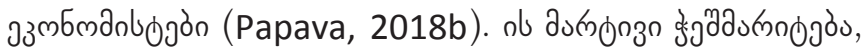

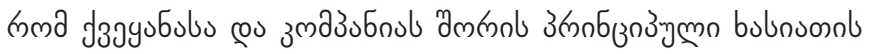

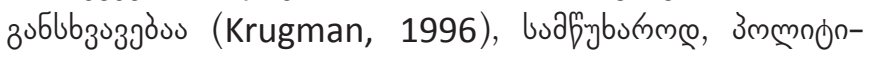

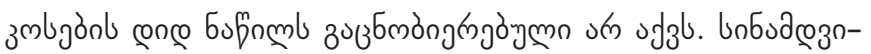

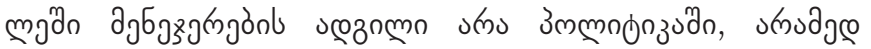

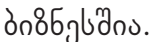

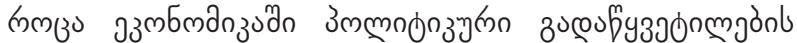
an

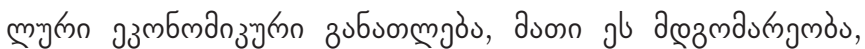

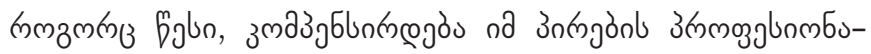

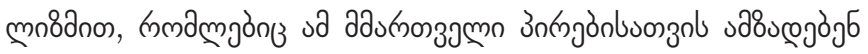

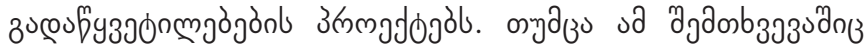

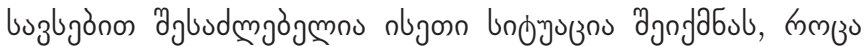

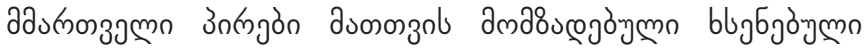

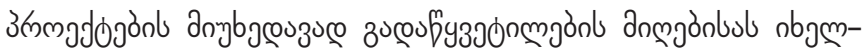

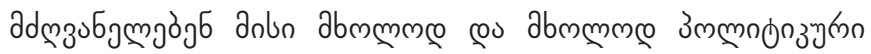

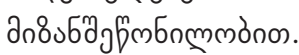

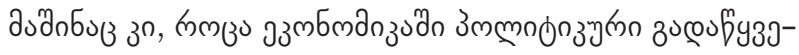

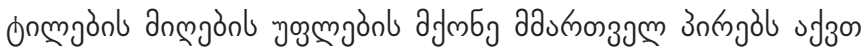

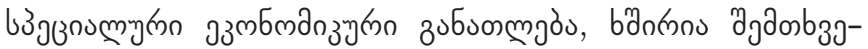

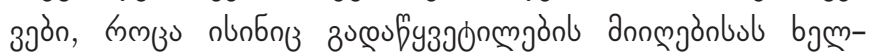

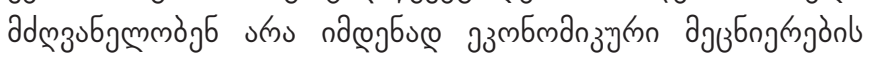

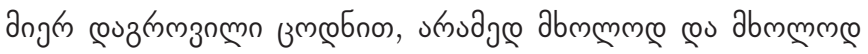

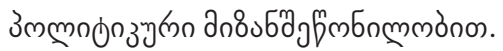

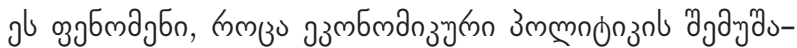

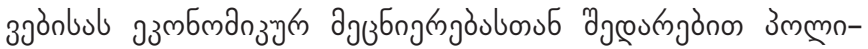

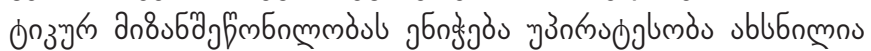

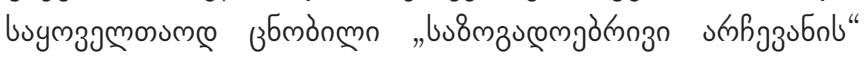
ogmknnob angr (Buchanan, Tullock, 1962).

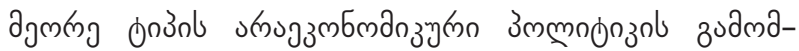

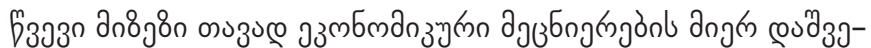

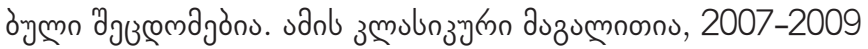

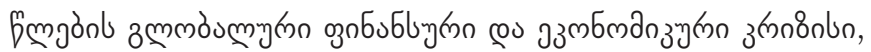

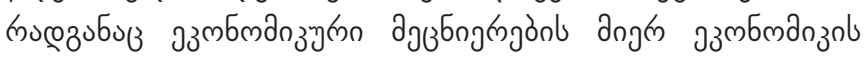

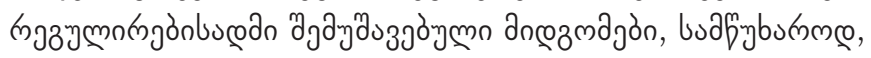

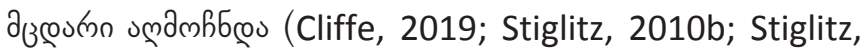
2010a).

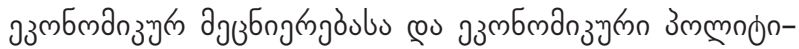

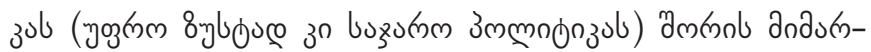

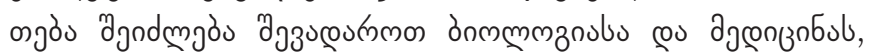

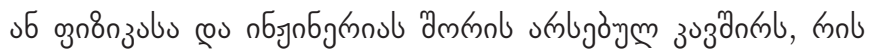

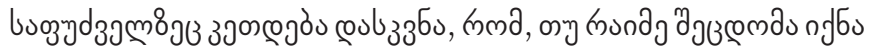
cos

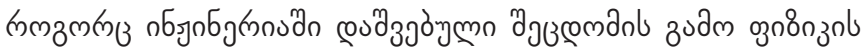

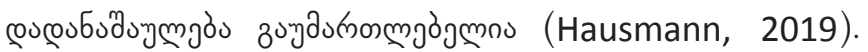

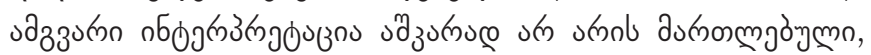
kuюgus

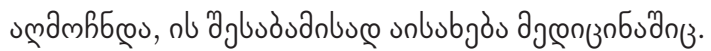

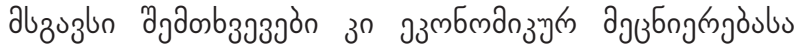

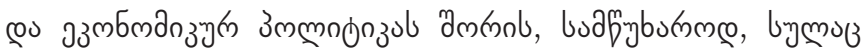

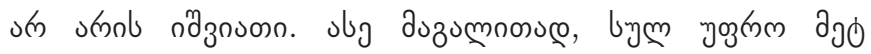

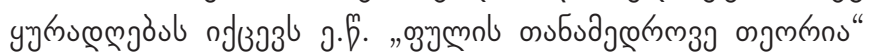

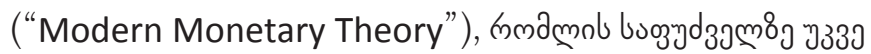

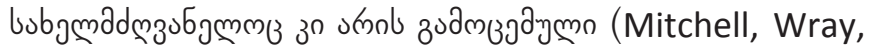

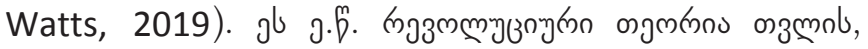




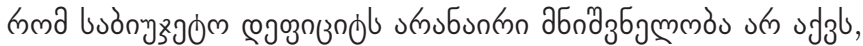

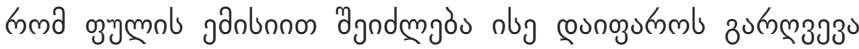

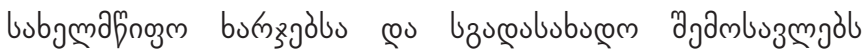

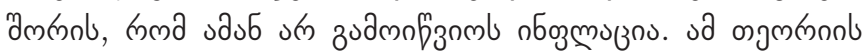

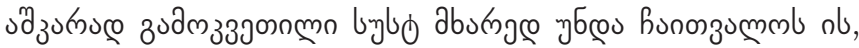

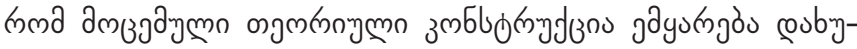

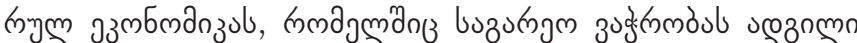

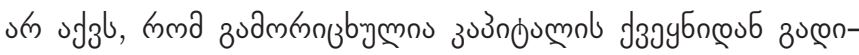

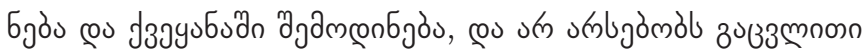

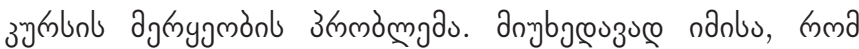

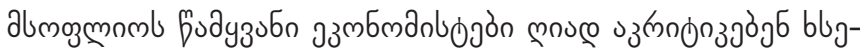

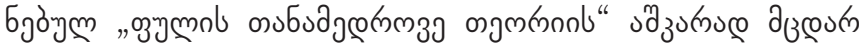
зmblofingdznol (Krugman, 2019a, 2019b; Rogoff, 2019),

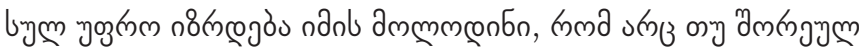

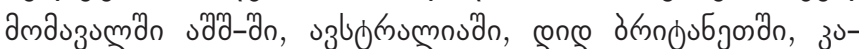
Бucoudn, эз з

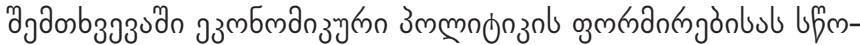

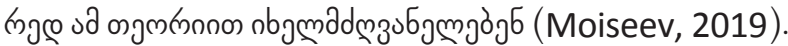

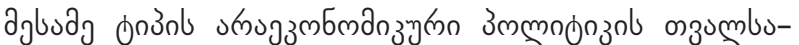

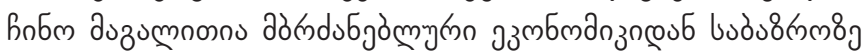

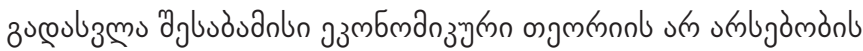

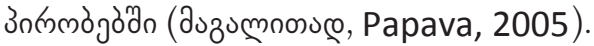

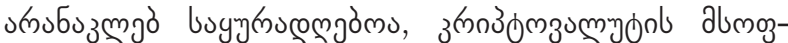

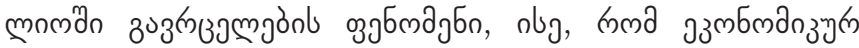

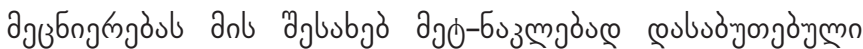

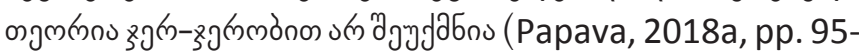

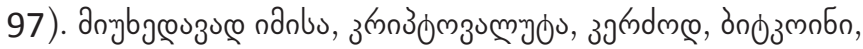

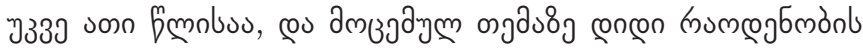

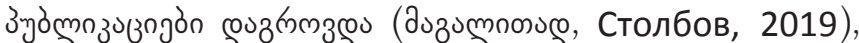

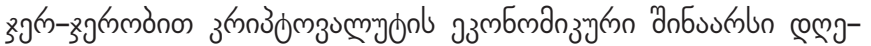

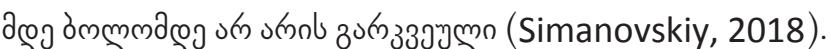

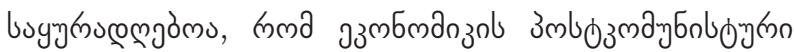

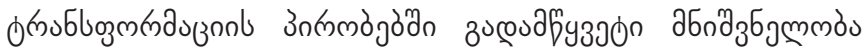

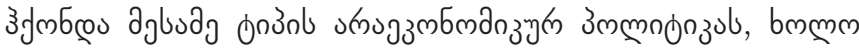

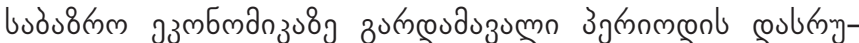

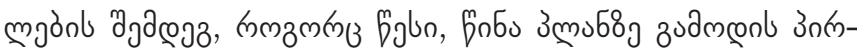

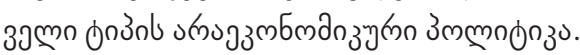

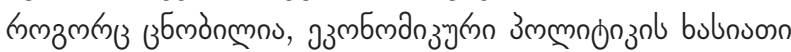

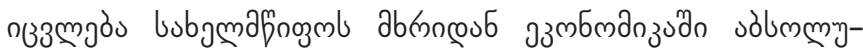

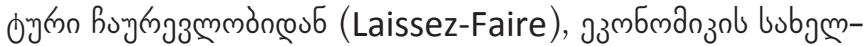

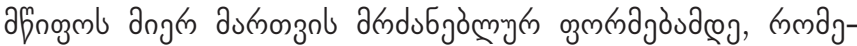

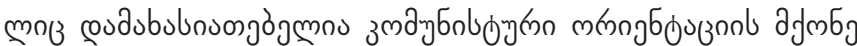

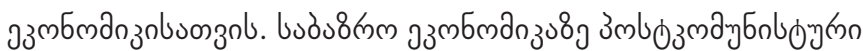

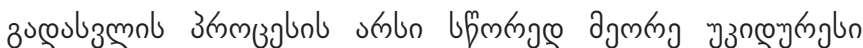

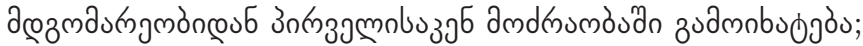

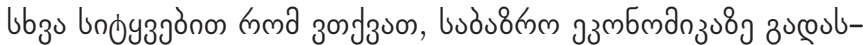

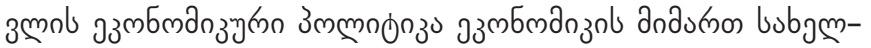

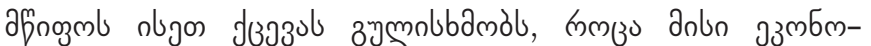

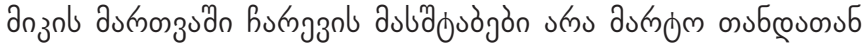

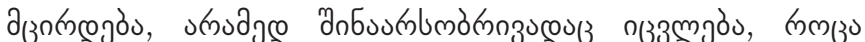

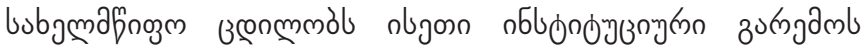

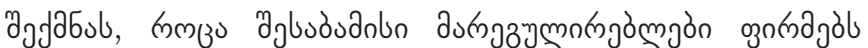

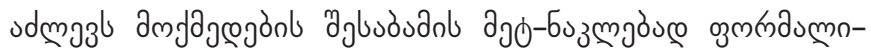
8jöng hombell.

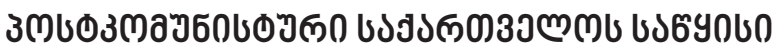

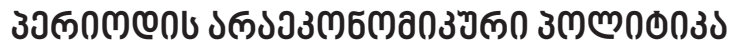

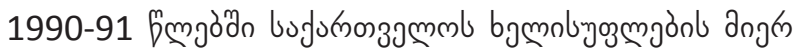

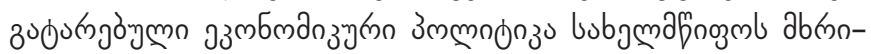

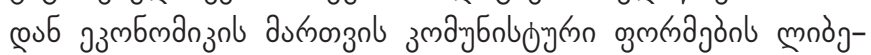

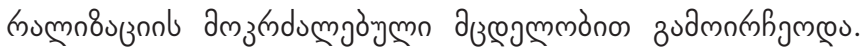

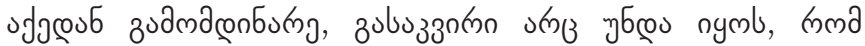

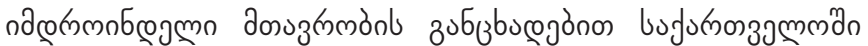

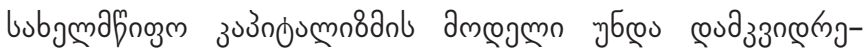

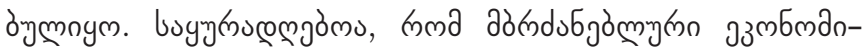

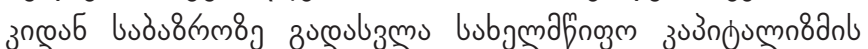

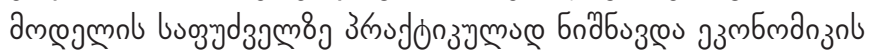

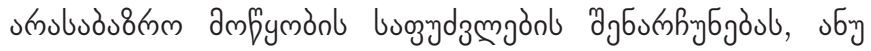

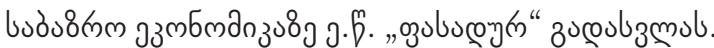

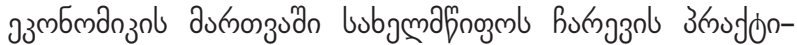

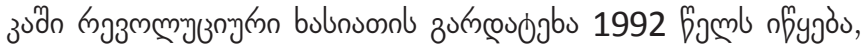

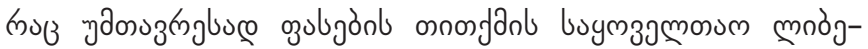

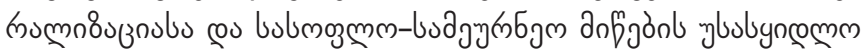

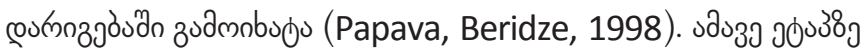

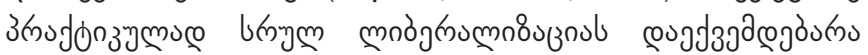

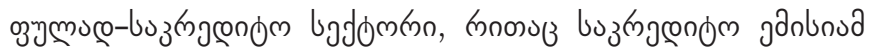

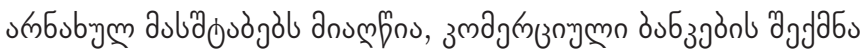

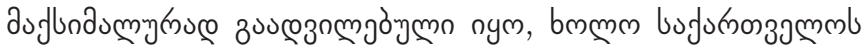

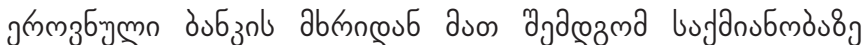

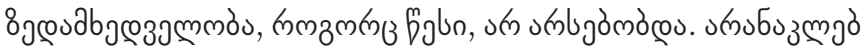

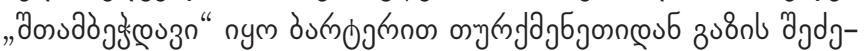

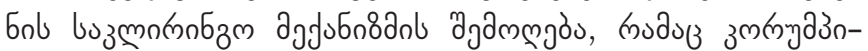

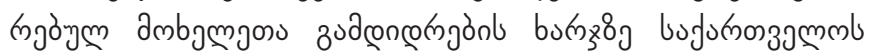

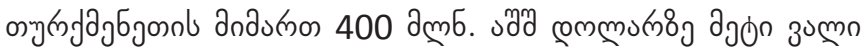

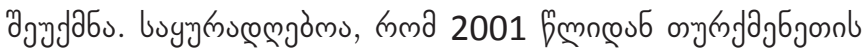

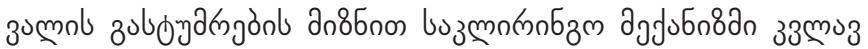

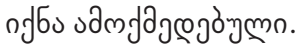

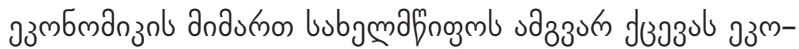

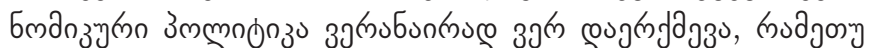

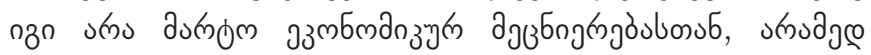

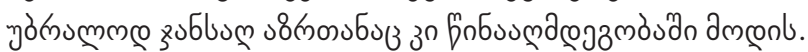

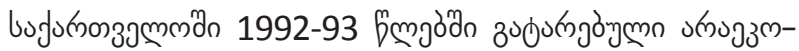

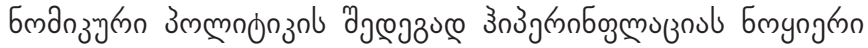

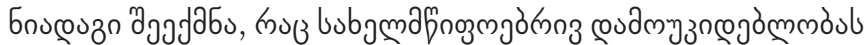

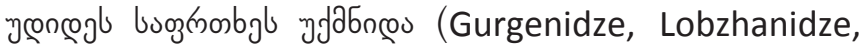
Onoprishvili, 1994). 


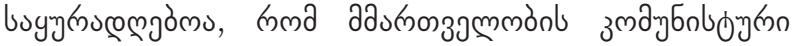

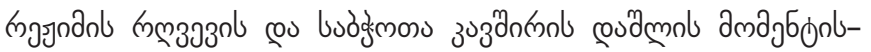

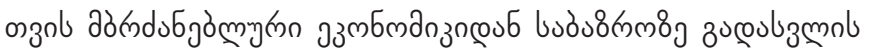

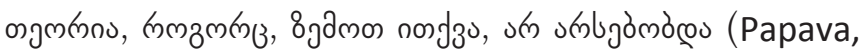

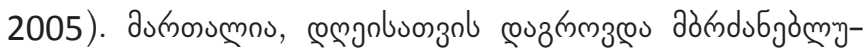

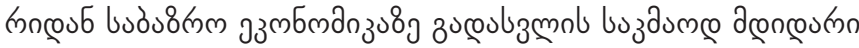

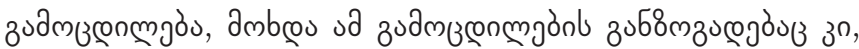

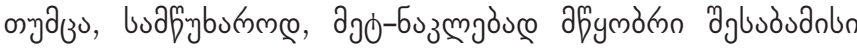

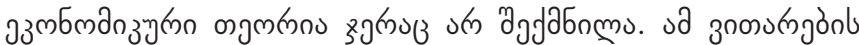

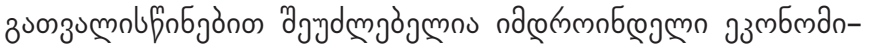

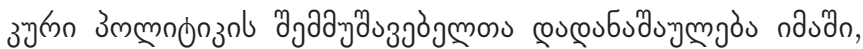

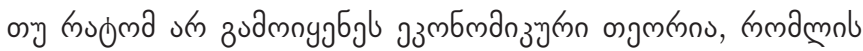

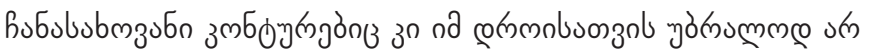

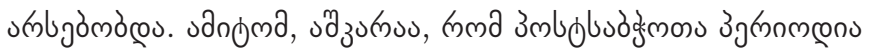

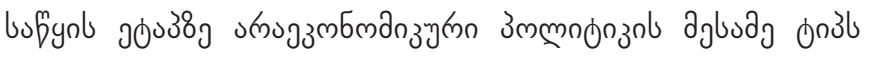

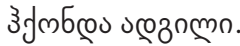

bufurnoszamman 1992 pmonb coubufynban counfym krugon-

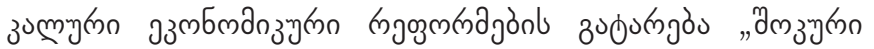

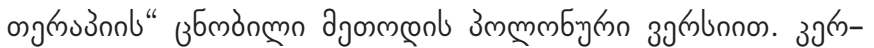

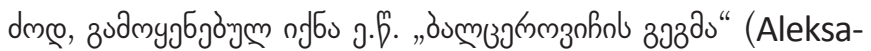

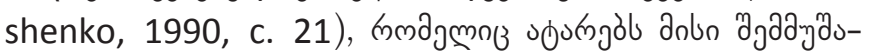

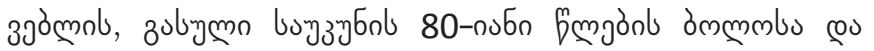

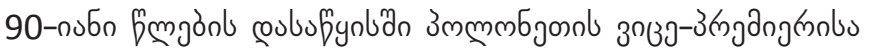

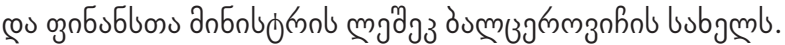

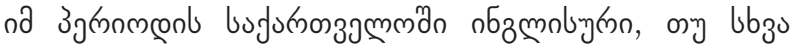

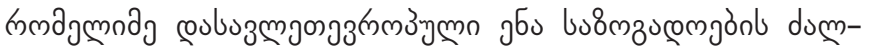

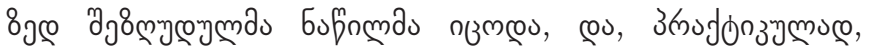

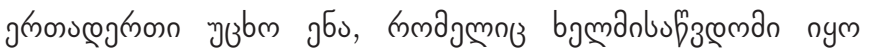

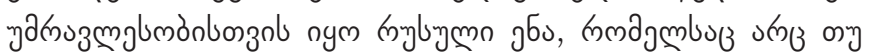

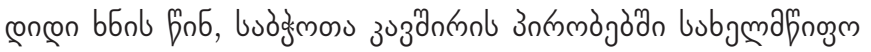

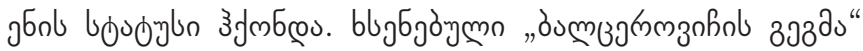

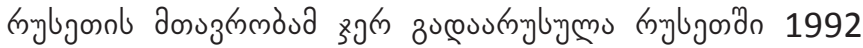

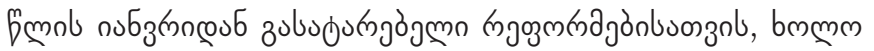

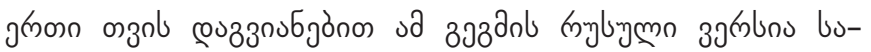

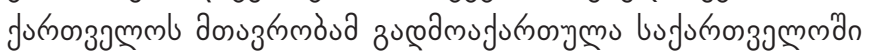

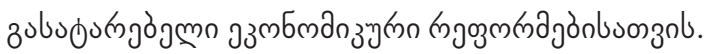

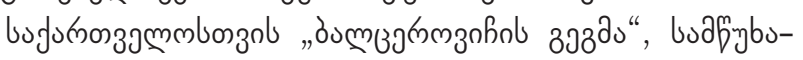

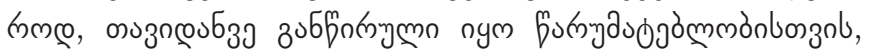

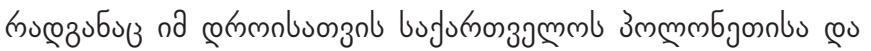

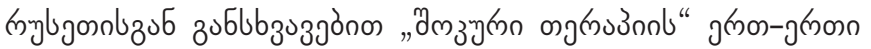

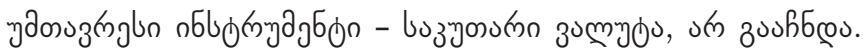

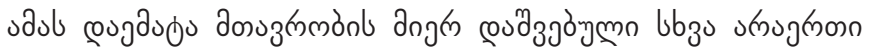

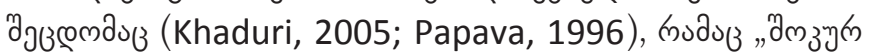

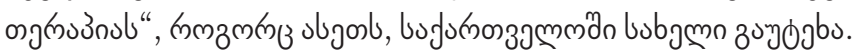

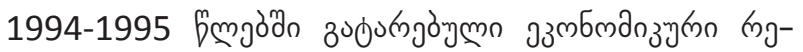

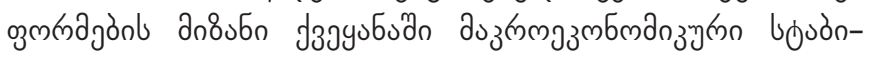

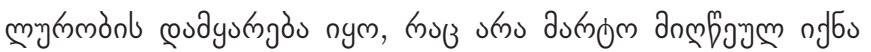

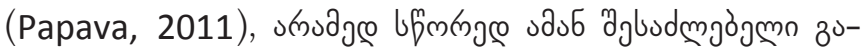

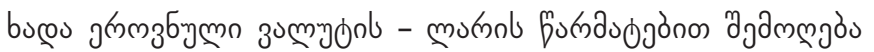
(Kakulia, 2008).

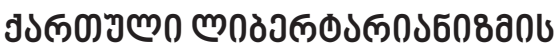

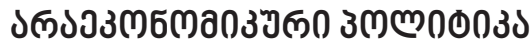

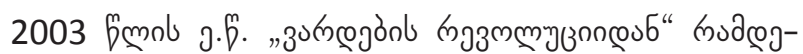

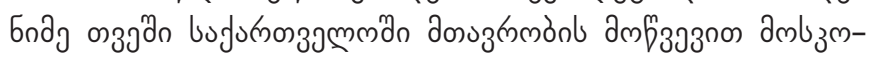

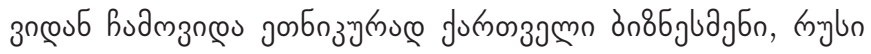

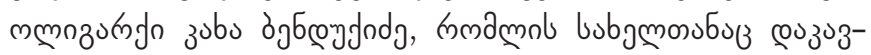

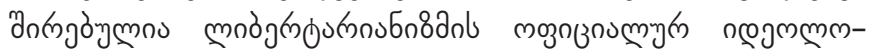

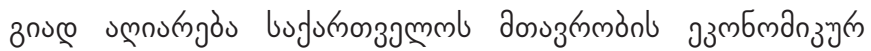

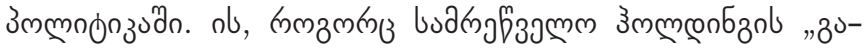

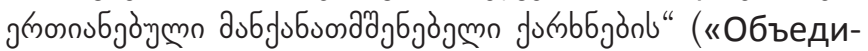
ненные машиностроительные заводы») 356 grg-

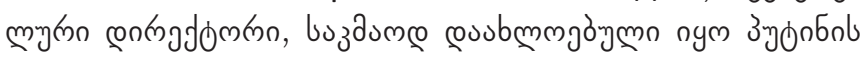

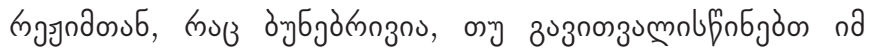

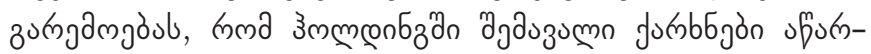

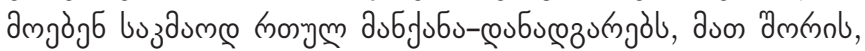

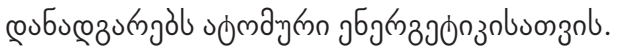

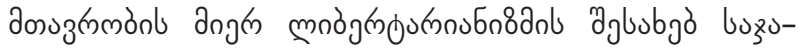

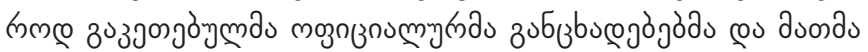

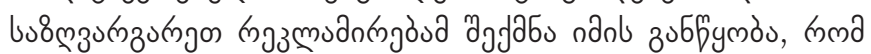

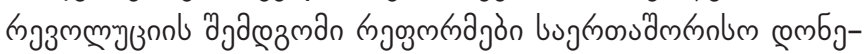

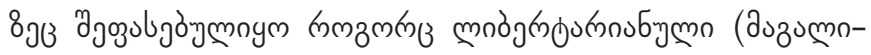
๓u(e, ESI, 2010; Gilauri, 2017; Udensiva-Brenner, 2010; Burakova, 2011).

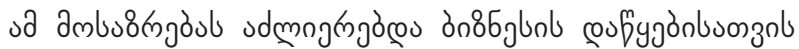

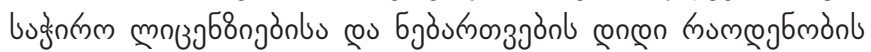

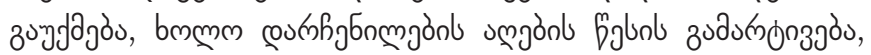

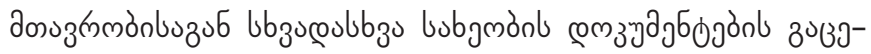

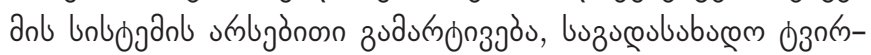

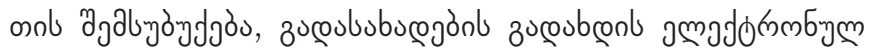

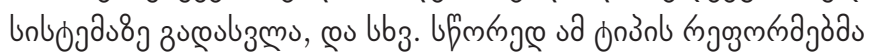

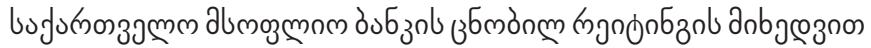

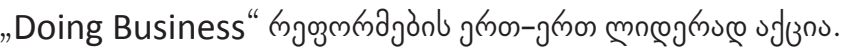

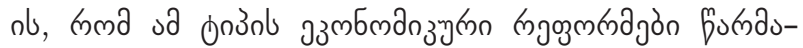

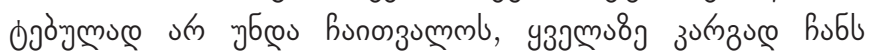

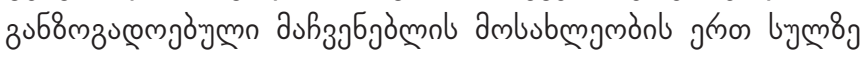

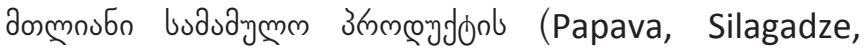

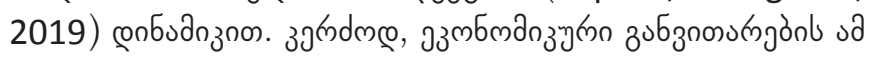

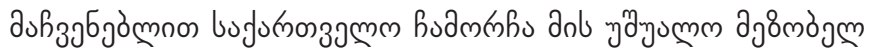

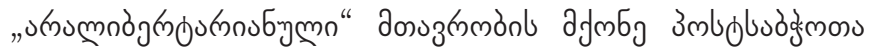

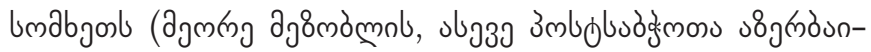

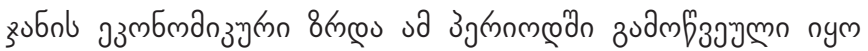

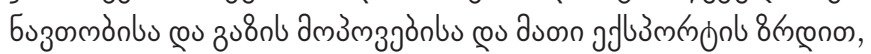

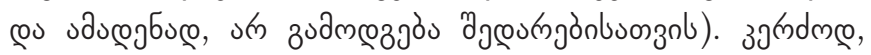

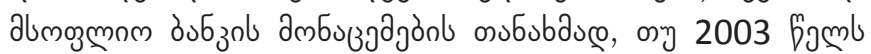

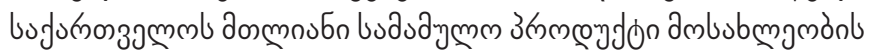

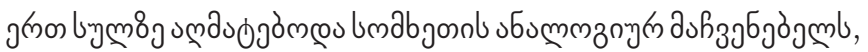

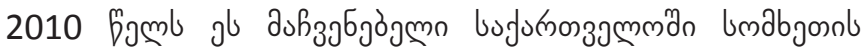

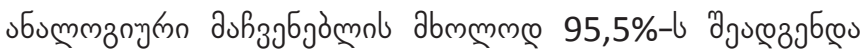
(WB, 2019). 


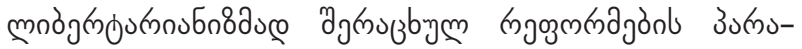

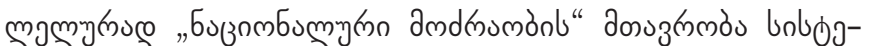

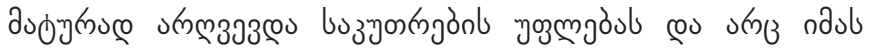

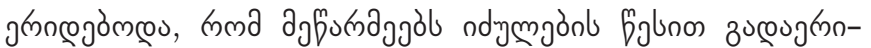

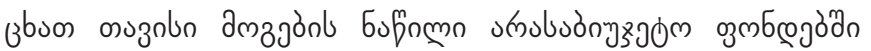

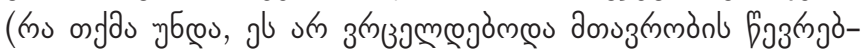

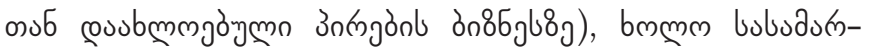

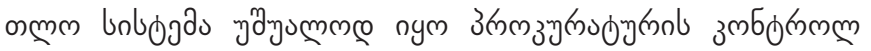

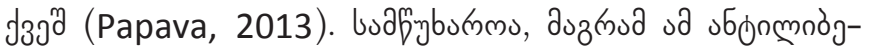

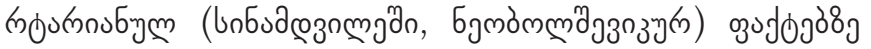

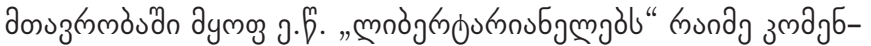

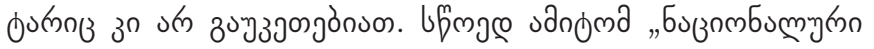

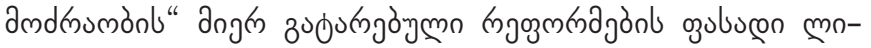

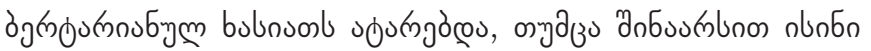

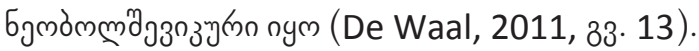

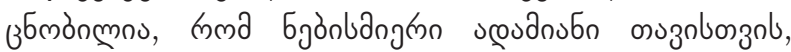

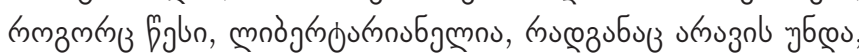

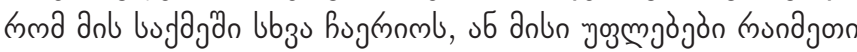

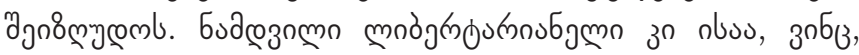

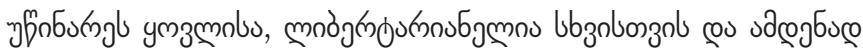
nl 8mzugougous mod̀gmounnobjmos.

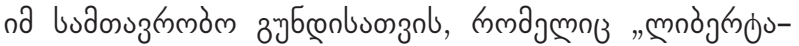

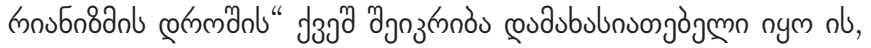

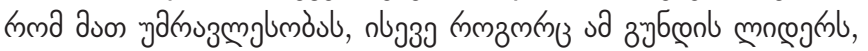

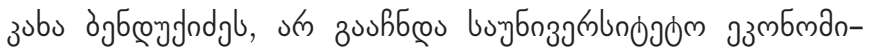

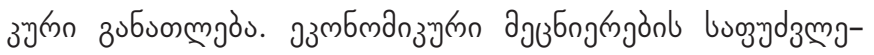

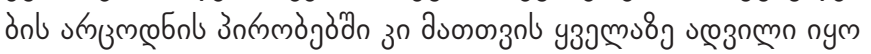

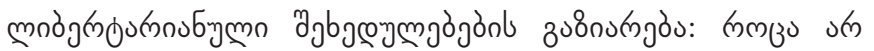

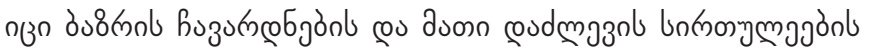

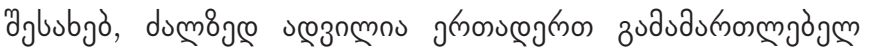

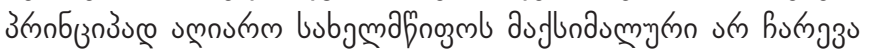
उзмбмдаз зेवे.

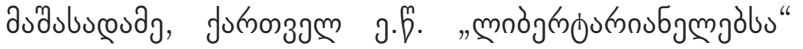

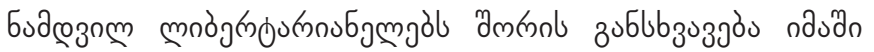

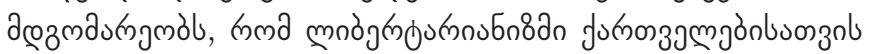

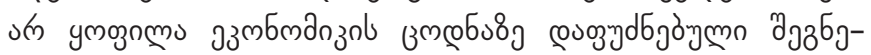

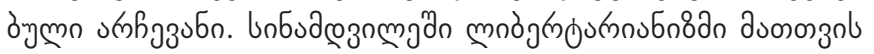

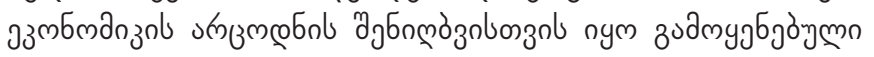
(Papava, Taphladze, 2015).

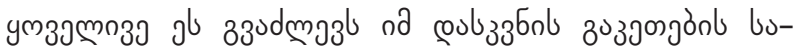

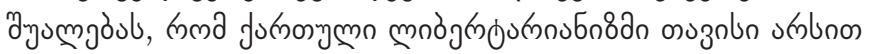

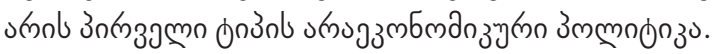

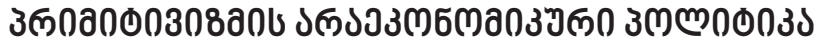

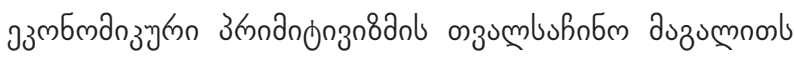

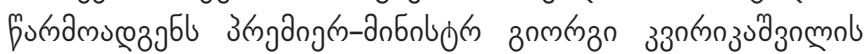

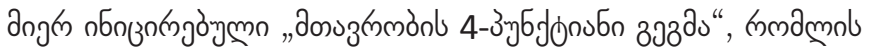

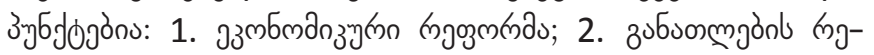

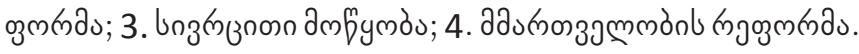

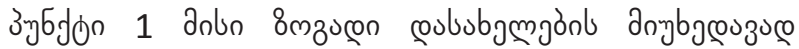

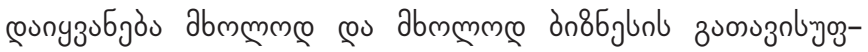

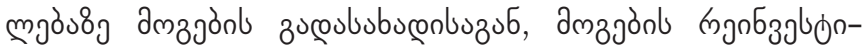

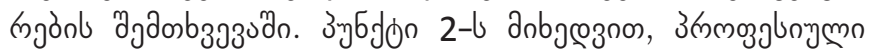

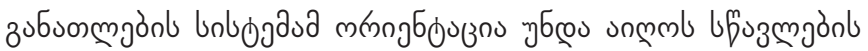

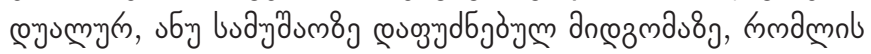

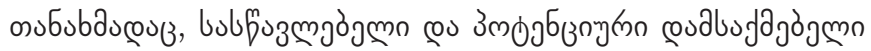

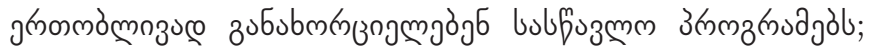

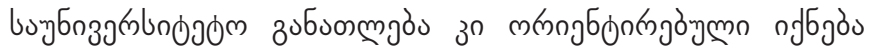

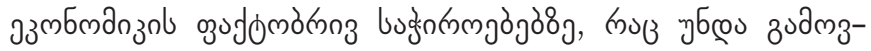

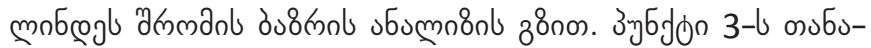

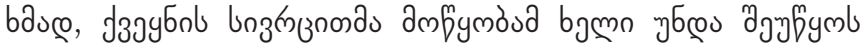

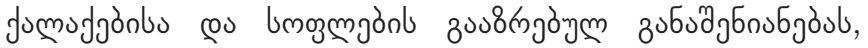

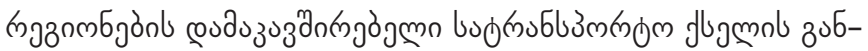

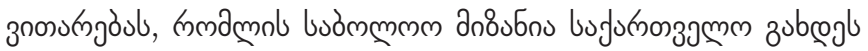

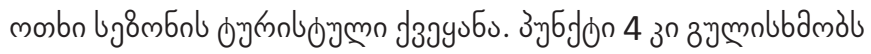

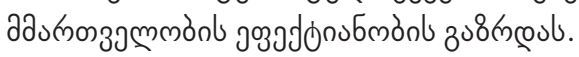

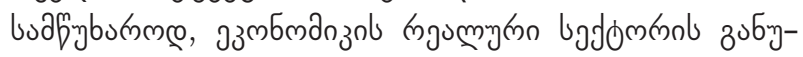

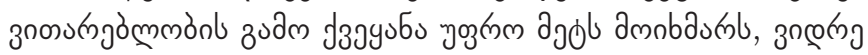

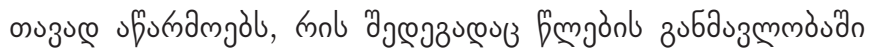

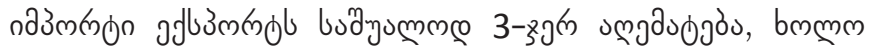

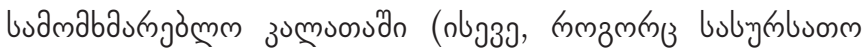

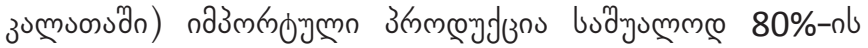

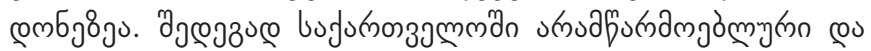

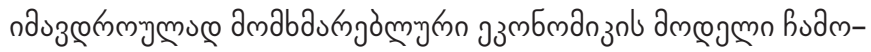
yumnò̉ou (Papava, 2015).

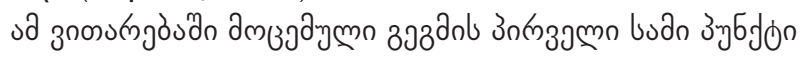

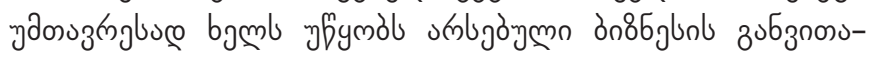

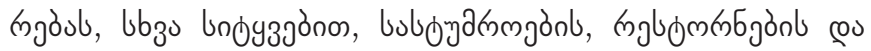

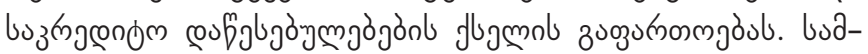

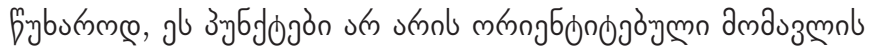

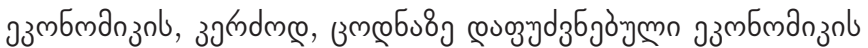

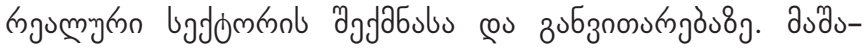

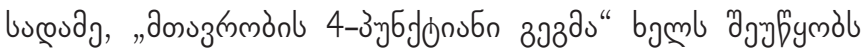

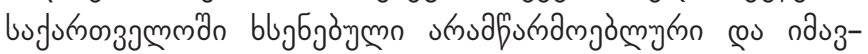

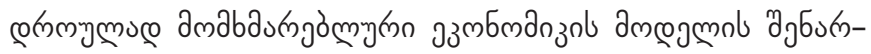

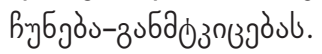

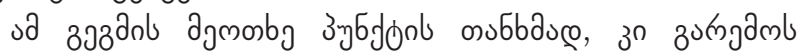

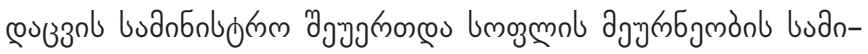

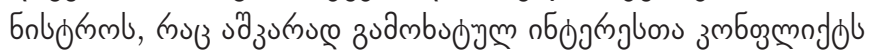

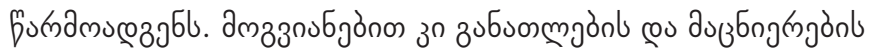

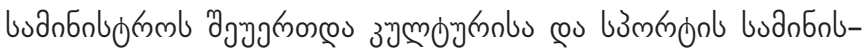

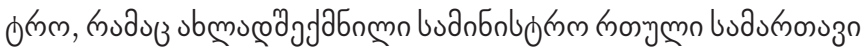

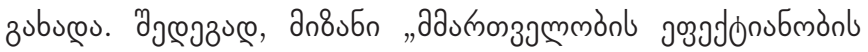

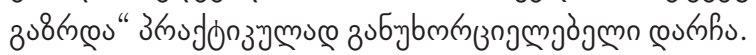

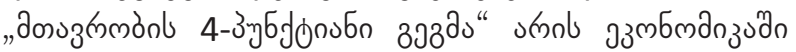

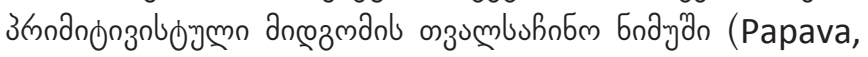

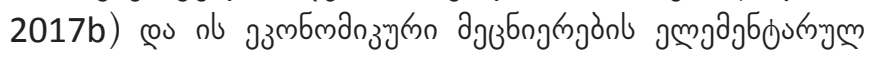

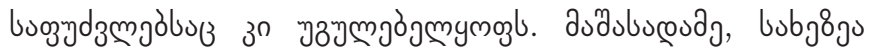

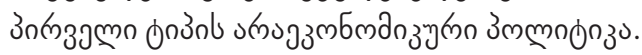

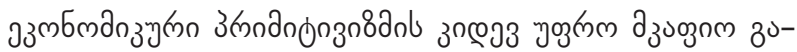




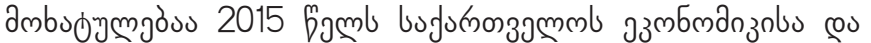

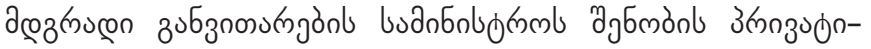

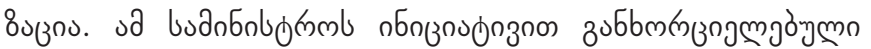

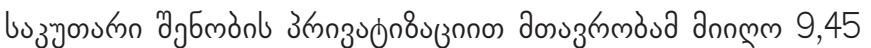

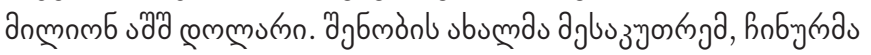

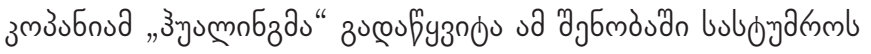
3ubl65.

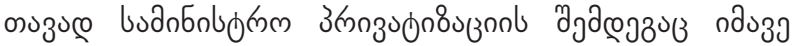

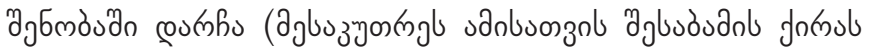

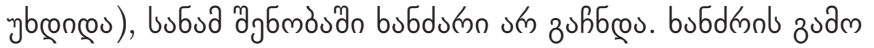

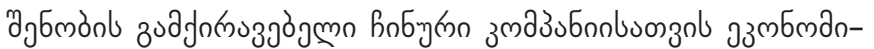

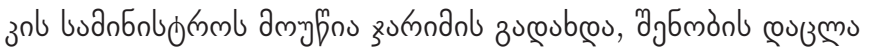

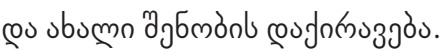

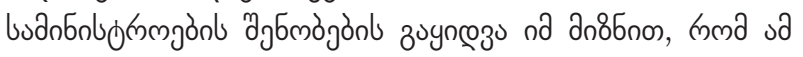

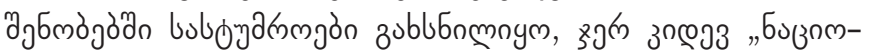

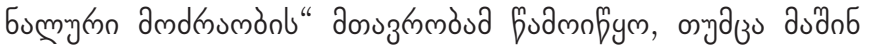

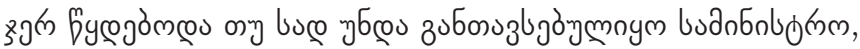

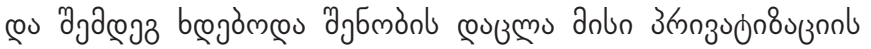

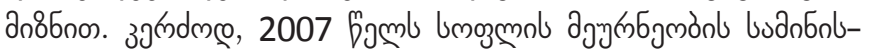

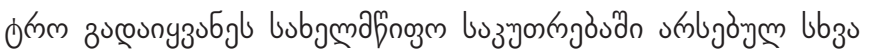

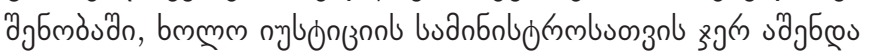

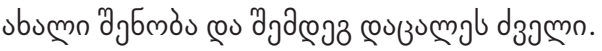

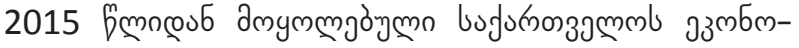

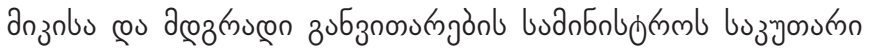
चुб

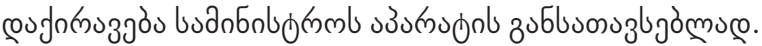

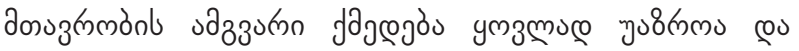

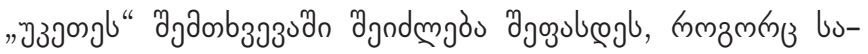

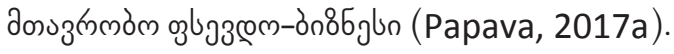

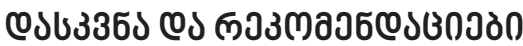

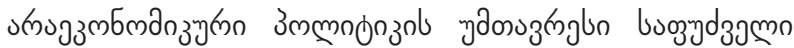
s

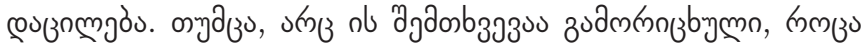

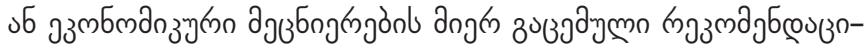

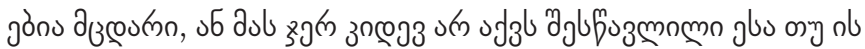

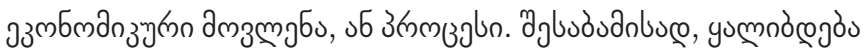

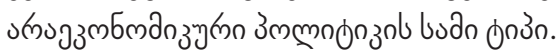

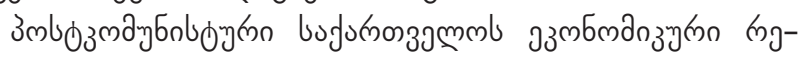

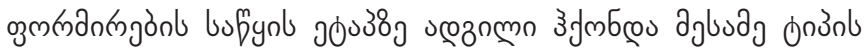

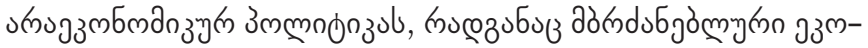

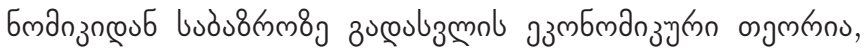

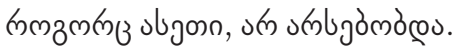

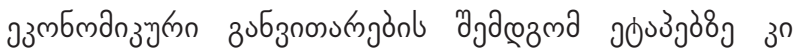

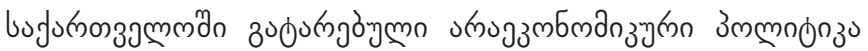

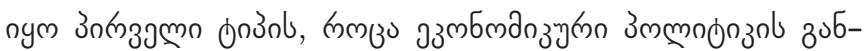

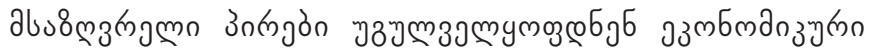

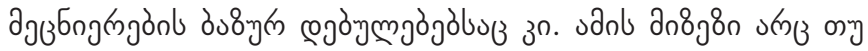

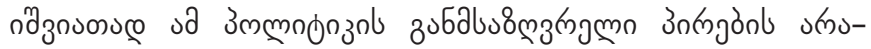
उங́mogglnombumnoban nym.

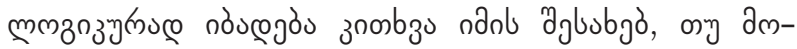

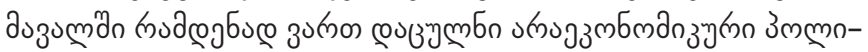

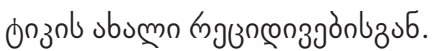

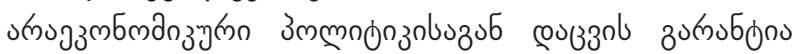

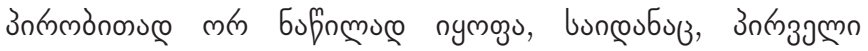

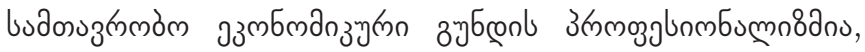

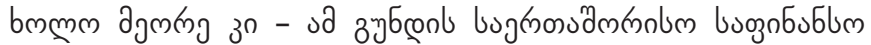

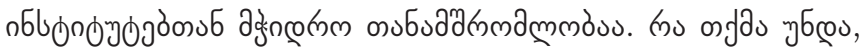

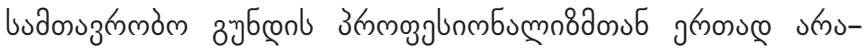

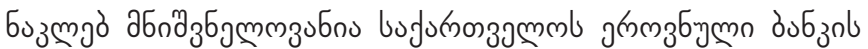

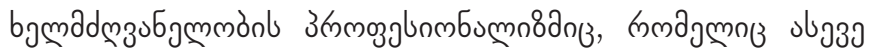

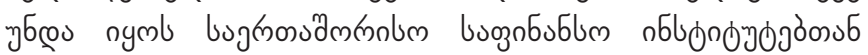

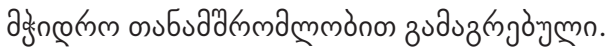

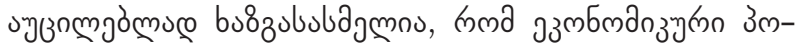

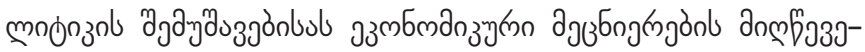

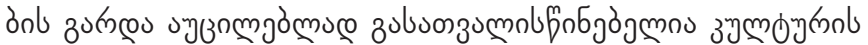
oुगJjmmn (Bedianashvili, 2018a, 2018b; Tambovtsev,

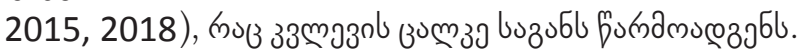

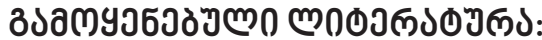

1. Aleksashenko C. (1990). Economic Reform: Polish Way [Ekonomicheskaja reforma: polskii put] " Mirovaja ekonomika i mezhdunarodnye otnoshenija [World Economy and International Relations] № 7, pp. 19-28. (In Russian).

2. Bedianashvili G. (2018a). "Knowledge Economy, Innovative Entrepreneurial Policy and Culture: Systemic View." In: Materials of the International Scientific-Practical Conference: "Modern tendencies of development of economy and economic science." Tbilisi: Paata Gugushvili Institute of Economics Publishing House, pp. 28-32.

3. Bedianashvili G. (2018b). Culture as a Factor of Knowledge Economics with Paradigmatic Changes in Systemic Institutional Context [kultura rogorts tsodnis ekonomikis phormirebis phaqtori paradigmuli tsvlilebebis sistemur-institutsiuri konteqstit] globalizatsia da biznesi [Globalization and Business] № 6, pp. 58-66. (In Georgian).

4. Bénassy-Quéré A., Coeuré B., Jacquet P., Pisani-Ferry J. (2016). Economic Policy: Theory and Practice [ekonomikuri politika: teoria da praqtika] Tbilisi: Ivane Javakhishvili Tbilisi State University Publishing House. (In Georgian).

5. Brown M. B. (1995). Models in Political Economy. A Guide to the Arguments. London: Penguin Books. 
6. Buchanan J. M., Tullock G. (1962). The Calculus of Consent: Logical Foundations of Constitutional Democracy. Ann Arbor: University of Michigan Press, http://www.econlib.org/library/Buchanan/buchCv3.html.

7. Burakova L. (2011). Why at Georgia it Turned Out [Pochemu u Gruzii poluchilos]. Moscow: United Press. (In Russian).

8. Cliffe M. (2019). What Economists Still Need to Learn. Project Syndicate, September 9, https://www.project-syndicate. org/commentary/macroeconomic-models-three-lessons-2008-crisis-by-mark-cliffe-2019-09?utm_source=Project +Syndicate+Newsletter\&utm_campaign=aabf9c90ac-sunday_newsletter_15_9_2019\&utm_medium=email\&utm_ term=0_73bad5b7d8-aabf9c90ac-93567601\&mc_cid=aabf9c90ac\&mc_eid=e9fb6cbcc0.

9. De Waal T. (2011). Georgia's Choices: Charting a Future in Uncertain Times [sakartvelos archevani: momavlis dagegmva gaurkvevlobis periodshi]. Washington, D.C.: Carnegie Endowment, (In Georgian).

10. https://carnegieendowment.org/files/georgias_choices_georgian.pdf. ESI (2010). Reinventing Georgia: The Story of a Libertarian Revolution. Berlin-Brussels-Istanbul: European Stability Initiative, https://www.esiweb.org/index. php?lang=en\&id=322\&debate_ID=3.

11. Gilauri N. (2017). Practical Economics: Economic Transformation and Government Reform in Georgia 2004-2012. Cham: Palgrave Macmillan.

12. Gurgenidze L., Lobzhanidze M., Onoprishvili D. (1994). “Georgia: From Planning to Hyperinflation.” Communist Economies \& Economic Transformation, Vol. 6, No. 2, pp. 259-289.

13. Hausmann R. (2019). "Don't Blame Economics, Blame Public Policy." Project Syndicate, September 1, https://www. project-syndicate.org/commentary/blame-public-policy-not-economics-by-ricardo-hausmann-2019-08?utm_source=Pr oject+Syndicate+Newsletter\&utm_campaign=d58b1700af-sunday_newsletter_8_9_2019\&utm_medium=email\&utm_ term=0_73bad5b7d8-d58b1700af-93567601\&mc_cid=d58b1700af\&mc_eid=e9fb6cbcc0.

14. Kakulia M. (2008). "Before and After the Introduction of the Lari: Georgian National Currency in Retrospect." In: Ismailov E. M., ed. Central Eurasia: National Currencies. Stockholm: CA\&CC Press, pp. 188-200.

15. Khaduri N. (2005). "Mistakes Made in Conducting Economic Reforms in Postcommunist Georgia." Problems of Economic Transition, Vol. 48, No. 4, pp. 18-29.

16. Krugman P. (1996). “A Country Is Not a Company." Harvard Business Review, January-February, https://hbr.org/1996/01/ a-country-is-not-a-company.

17. Krugman P. (2019a). "Running on MMT (Wonkish)." The New York Times, 25 February, https://www.nytimes. com/2019/02/25/opinion/running-on-mmt-wonkish.html.

18. Krugman P. (2019b). "What's wrong with functional finance? (Wonkish)." The New York Times, 12 February, https:// www.nytimes.com/2019/02/12/opinion/whats-wrong-with-functional-finance-wonkish.html.

19. Mitchell W., Wray R., Watts M. (2019). Macroeconomics. London: Macmillan Education.

20. Moiseev R. (2019). Hype Around (Non)Monetary (Non)Theory [Khaip vokrug (ne)denezhnoi (ne)teorii]. Voprosy ekonomiki [Economic Issues], No. 9, pp. 112-122. (In Russian)

21. Papava V. (1996). "The Georgian Economy: From 'Shock Therapy' to 'Social Promotion'." Communist Economies \& Economic Transformation, Vol. 8, No. 2, pp. 251-267.

22. Papava V. (2002). Political Economy of the Post-Communist Capitalism and its Application for Georgian Economy [poskomunisturi kapitalizmis politikuri ekonomia da sakartvelos ekonomika]. Tbilisi: "PDP." (In Georgian).

23. Papava V. (2005). "On the Theory of Post-Communist Economic Transition to Market." International Journal of Social Economics, Vol. 32, No. 1/2, pp. 77-97.

24. Papava V. (2011). "On the First-Generation Post-Communist Reforms of Georgia's Economy (A Retrospective Analysis)." The Caucasus \& Globalization, Vol. 5, Issue 3-4, pp. 20-30.

25. Papava V. (2012). Economic Reforms in Post-Communist Georgia: Twenty Years After. New York: Nova Science Publishers.

26. Papava V. (2013). "Economic Achievements of Postrevolutionary Georgia: Myths and Reality." Problems of Economic Transition, Vol. 56, No. 2, pp. 51-65.

27. Papava V. (2015). "Necroeconomics of Post-Soviet Post-Industrialism and the Model of Economic Development of Georgia and Russia." Journal of Business and Economics, Vol. 6, No. 5, pp. 978-979, www.academicstar.us/UploadFile/ Picture/2015-7/20157313847837.pdf.

28. Papava V. (2017a). “Features of Governmental ‘Business' in Post-Soviet Georgia.” Eurasia Review. Journal of Analysis and News, June 27, https://www.eurasiareview.com/27062017-features-of-governmental-business-in-post-soviet-georgia-oped/.

29. Papava V. (2017b). "Primitivism as a Trait of Georgia's Modern Economic Policy." The Central Asia-Caucasus Analyst, May 4,http://cacianalyst.org/publications/analytical-articles/item/13444-primitivism-as-a-trait-of-georgia\%E2\%80\%99smodern-economic-policy.html.

30. Papava V. (2018a). "The Economics in Crisis and the Main Directions for Transformation of Economic Science." Transformations, No. 3-4 (98-99), pp. 90-108, http://www.e-transformacje.com/archiwum/transformacje_3-4_2018. pdf\#page=96.

31. Papava V. (2018b). "Why Georgia Needs Economists." Rondeli Blog, October 12, https://www.gfsis.org/blog/view/871.

32. Papava V., Beridze T. (1998). Economic Reforms in Georgia [Ekonomicheskie reformy v Gruzii] Rossiiskii ekonomicheskii zhurnal [Russian Economic Journal,] №1, pp.58-64. (In Russian). 
33. Papava V., Silagadze A. (2019). On the Georgian Name of One Key Economic Term “Gross Domestic Product [erti sakvandzo ekonomikuri terminis - "Gross Domestic Product"-is kartuli sakheltsodebis shesaxeb] ekonomika da biznesi [Economics and Business,] No. 1, pp. 180-182. (In Georgian).

34. Papava V., Taphladze T. (2015). "Pseudo-Libertarianism in Post-Revolutionary Georgia." Bulletin of the Georgian National Academy of Sciences, Vol. 9, No. 2, pp. 150-153.

35. Pearce D. W., ed. (1997). Slovar' sovremennoi ekonomicheskoi teorii Makmillana [Macmillan Dictionary of Modern Economics.] Moscow: INFRA-M. (In Russian).

36. Rogoff K. (2019). "Modern monetary nonsense." Project Syndicate, March 4, https://www.project-syndicate.org/ commentary/federal-reserve-modern-monetary-theory-dangers-by-kenneth-rogoff-2019-03?barrier=accesspaylog.

37. Simanovskiy A. Yu. (2018). "K voporosu ob ekonomicheskoi prirode kriptovaljuty [On the Issue of Crypto-Currency Economic Nature.]" Voprosy ekonomiki [Economic Issues,] № 9, pp. 132-142. (In Russian).

38. Stiglitz J. (2010a). "Ekonomisty vinovaty v krizise, no est' shans ispravit' delo [Economists are Guilty in Crisis, but there is a Chance to Correct the Situation.]" Institut problem predprinimatel'stva [Institute of Problems of Entrepreneurship,] October 5, https://www.ippnou.ru/article.php?idarticle=008769. (In Russian).

39. Stiglitz J. (2010b). "Needed: A New Economic Paradigm." Financial Times, August 20, https://www.ft.com/content/ d5108f90-abc2-11df-9f02-00144feabdc0.

40. Stolbov M. I. (2019). "K desjatiletiju rynka kriptovaljut: tekushchee sostojanie i perspektivy [The 10th Anniversary of the Cryptocurrency Market: Its Current State and Prospects."] Voprosy ekonomiki [Economic Issues,] № 5, pp. 136-148. (In Russian).

41. Tambovtsev V. (2015). "Mif o "kulturnom kode" v ekonomicheskikh isskedovanijakh [The Myth of the "Culture Code" in Economic Research."] Voprosy ekonomiki [Economic Issues,] No. 12, pp. 85-106. (In Russian).

42. Tambovtsev V. L. (2018). "Innovatsii i kultura: vazhnost' metodologii analiza" ["Innovations and Culture: Importance of the Analysis Methodology."] Voprosy ekonomiki [Economic Issues], No. 9, pp. 70-94. (In Russian).

43. Udensiva-Brenner M. (2010). "Kakha Bendukidze Analyzes Georgia's Economic Strategy: How Georgia Handled Its Economy After the War and the Economic Crisis." Harriman Institute, April 7, https://harriman.columbia.edu/event/ kakha-bendukidze-analyzes-georgia\%E2\%80\%99s-economic-strategy-how-georgia-handled-its-economy-after.

44. WB (2019). "GDP per capita (current US\$)." The World Bank, https://data.worldbank.org/indicator/ny.gdp.pcap. cd?end=2003\&start=1960. 


\title{
POLITICIZATION OF THE ECONOMY AND NON-ECONOMIC POLICY (EXPERIENCE OF POST-COMMUNIST GEORGIA)
}

\author{
VLADIMER PAPAVA \\ Professor, \\ Ivane Javakhishvili Tbilisi State University, \\ Principal Research Fellow, Paata Gugushvili Institute of Economics, Georgia \\ vladimer.papava@tsu.ge
}

KEYWORDS: ECONOMIC POLICY, ECONOMIC SCIENCE, POLITICIZATION OF ECONOMICS, ECONOMIC REFORMS, HIGH-PROFILE ECONOMISTS, INTERNATIONAL FINANCIAL INSTITUTIONS.

For citation: Papava, V. (2019). Politicization of the Economy and Non-Economic Policy (Experience of Post-Communist Georgia), Globalization And Business, №8, pp. 13-21. https://doi.org/10.35945/gb.2019.08.001

\section{SUMMARY}

It is not so uncommon when the main deterring factor of economic growth is its politicization. As a matter of fact, economic policy is a part of governmental policy and this means that the "politicization of the economy" is practically unavoidable. The problem is when the conditioning of the "politicization of the economy" is the type of economic policy that is not based on the achievements of economic science. If economic policy is not only distant from economic science but, on some occasions even contradicts it with its elementary provisions, then this type of economic policy held by a government should be called "non-economic policy." Among the causes of "non-economic policy," special attention must be paid to problems in the existing mistakes in economics, in the non-professional economic team of a government and in the wrong recommendations given to a government by international financial institutions. Post-communist Georgia's experience gives us noticeable examples when different governments held 'non-economic policies' which negatively impacted the country's development. As a result of successful economic policies, economic reforms were held which subsequently combatted hyperinflation in the 1990s, achieved macroeconomic stability and established the Georgian lari (GEL) as the national currency following currency reform. Relevant reforms were completely based on economics. The main problem is the rapprochement of economic policy to economic science. Achievement of this is only possible in the case when economic policy is elaborated by highly professional economists. 